\title{
ENCHANCING THE USE OF BIDSS/CI SYSTEMS: A PROPOSED FRAMEWORK
}

\author{
Monica C. Holmes, Central Michigan University, monica.c.holmes@cmich.edu \\ Roger L. Hayen, Central Michigan University, roger.l.hayen@cmich.edu
}

Jie Zeng, Cleveland Clinic Foundation, jzeng102@hotmail.com

\begin{abstract}
Nowadays applications for Business Intelligence (BI) and Decision Support Systems (DSS) have been adopted by multiple organizations. BIDSS have become one of the most significant tools for managers. Big corporations as well as small enterprises annually spend billions of dollars for BIDSS software in order to improve design, manufacturing, service, marketing, management, and financial decision-making. However, purchasing and installing BI/DSS software is not a guarantee of the effectiveness and efficiency of their implementation. This research effort examines several problems that companies face with the implementation of BIDSS. The purpose is to determine the characteristics of those problems and provide an innovative solution for them. A case study that illustrates an application of BI/DSS in a chemical corporation is included.
\end{abstract}

Keywords: Applications, BI, Business Intelligence, Chemical Industry, CI, Competitive Intelligence, DSS, Decision Support Systems.

\section{INTRODUCTION}

Existing Business Intelligence (BI) /Decision Support Systems (DSS) theoretical frameworks mainly focus on the analysis of information using various intelligent tools or software [1], IT infrastructure's deployment via equipping new IT technologies or hardware [6], and the arrangements of a company's resources emphasizing the issue of cost-effectiveness [8]. The implementation of a BI basically focuses on dealing with information using various intelligence tools or software to transform raw information into business knowledge; DSS mainly focuses on extracting, reporting and presenting aforementioned information to decision makers helping them accelerate the process of decision-making. Both are useful for a company to gain the competitive edge in everyday business processes. The purpose of this study is to integrate BI and DSS into one system based on a current BIDSS framework (see Figure 1), and to include aspects of Competitive Intelligence (CI).

\section{LITERATURE REVIEW}

A literature review, based on academic sources such as journals, magazines, libraries or online databases, describe the typical processes, workflow, and applications of BIDSS [4, 7, 9, 10, 12]. BIDSS is a combination of artificial intelligence, DSS, data mining, knowledge management, online analytical processing (OLAP) and web technologies with traditional personal productivity software tools [13]. It is also necessary to address the platform or infrastructure on which a BIDSS operates in order to deliver a clearer understanding of the components and technologies used to the readers. A typical BIDSS is divided into three levels: 1) the bottom level, which is the path of source data collected and the repository of data storage; 2) the middle level which consists of a wide variety of intelligence software or tools; 3 ) the top level, the user interface, in which the final report is viewed and delivered to users. A BIDSS must be deployed within an infrastructure or platform with the capabilities to implement the BIDSS process to support the range of applications best suited to every business requirement [11]. Below is a typical BIDSS platform or infrastructure with currently popular capabilities described below.

\section{Data warehouse}

The data warehouse is usually considered to be the bottom level and directly linked to a variety of information source paths. It keeps the records of an enterprise's past transactional and operational activities, gathering information from business environments. The database design favors data analysis and reporting in order to gain strategic insights and to facilitate decision making. It often holds large amounts of information which are sometimes subdivided into smaller logical units called dependent data marts $[14,16,17,18]$.

\section{OLAP}

Within the middle analytical level, the core of a BIDSS, Online AnaLytical Processing (OLAP) is a critical BIDSS component. It is a category of 
software technology and is the fundamental foundation for BIDSS solutions. It enables analysts, managers and executives to gain insight into data through fast, consistent, interactive access to a wide variety of possible views of information transformed from raw data to reflect the real dimensionality of the enterprise as understood by the user [19]. OLAP analysis becomes to truly powerful when combined with predictive analysis from data mining.

\section{Data Mining}

Similarly, within the middle analytical level, data mining is an analytic process designed to explore large amounts of data collected in search of consistent patterns or systematic relationships between variables. It then validates the findings by applying the detected patterns to new subsets of data. Data mining tools predict behaviors and future trends, allowing businesses to make proactive, knowledgedriven decisions. Data mining tools can answer business questions that traditionally were too time consuming to resolve $[6,20]$. It also is known as knowledge-discovery in databases (KDD).

\section{The user interface}

From the users' point of view, the interface is the only way to evaluate a system--whether or not it interacts effectively and efficiently with them. It is the most visible component of a working system. The interface is in charge of responding to the users' queries for business questions, to deliver the final reports to them through providing a portal or an entry to users to access and interact with the whole system. To make a BIDSS as effective as possible, people factors must be included when designing the interface. The user ability to use the system efficiently is directly linked to the limitation of his/her knowledge of the system [4].

Most of the existing data systems were designed using the user model based on his/her profession. However, a BIDSS model is not complete since each user reacts differently according to his needs and his working habits as well as his/her knowledge [9]. Thereby a customized model or interface designed specifically for the user is necessary to significantly enhance and improve their effective use of system operations in practice. This interface should be kept as simple or easy as it can be and should be suitable for different levels of users. In the rapid and everchanging business environment, the time needed to make a decision is critical to a company. Making sure that the right information delivered to right person at the right time is a vital for success of any BIDSS [1].
Few studies, however, have touched on the user or people factors in a BI/DSS. Yet the people or user factors are extremely important for utilizing a BIDSS effectively and efficiently as more and more organizations have realized [3]. This research analysis supports the re-integration of BI and DSS into a whole system based on a current BIDSS framework (Figure 1) and address in detail the basic principles and application of business intelligence decision support system as well as its effectiveness and impact in businesses. The analysis is presented by: (1) identifying the BIDSS vision; (2) determining the role and impact of people factors either in the process of information gathering or the performance effectiveness of the BIDSS systems; (3) evaluating methods of reporting and presenting the information in implementing the BIDSS; (4) distinguishing elements necessary for building a feasible BIDSS infrastructure and addressing its decision principle; and (5) security issue of BIDSS.

\section{METHODOLOGY AND DATA ANALYSIS}

This research is based on a case study of a large chemical company in the Midwestern region of the United States. Qualitative research is conducted with the use of interviews of critical personnel who use Competitive Intelligence (CI) tools and methodologies to enhance their decision making.

The research methodology is based on the current BIDSS model (Figure 1) which is explained in the previous section. However, certain features are missing from this particular framework. First of all, it needs a platform or interface for the final results to be presented or delivered to decision-makers. Secondly, there is no illustration of how information is collected from external sources such as marketplaces or competitors, data that is critical for including competitive advantages for a company. Thirdly, security controls seem to be taken for granted or ignored [15]. The authors plan to use the case study to evaluate if these features are necessary.

\section{Data collection}

Using the BIDSS framework in Figure 1, data is collected through interviews of personnel who work with BIDSS. Six people in two areas, IT and business, and three managerial levels-executive, middle-manager, and operational--are interviewed. Brief descriptions of the six individuals interviewed follow: 
1. A business area expert with more than 25 years experience in the polymers and chemical business;

2. A global commercial expert with more than 20 years in the analysis and prediction of market trends and competitors' actions;

3. An IT expert in global competitive intelligence;

4. A product sales representative with more than 10 years experience in the business;

5. A middle-level IT manager with over 30 years working experience in system design and more than 15 years experience in designing and implementing competitive intelligence solutions;

6. A system operator with more than 10 years experience.

The interview questions focus on the three issues identified in the current existing model-user interface, data source, and access portal for several reasons. Firstly, the authors believe that user

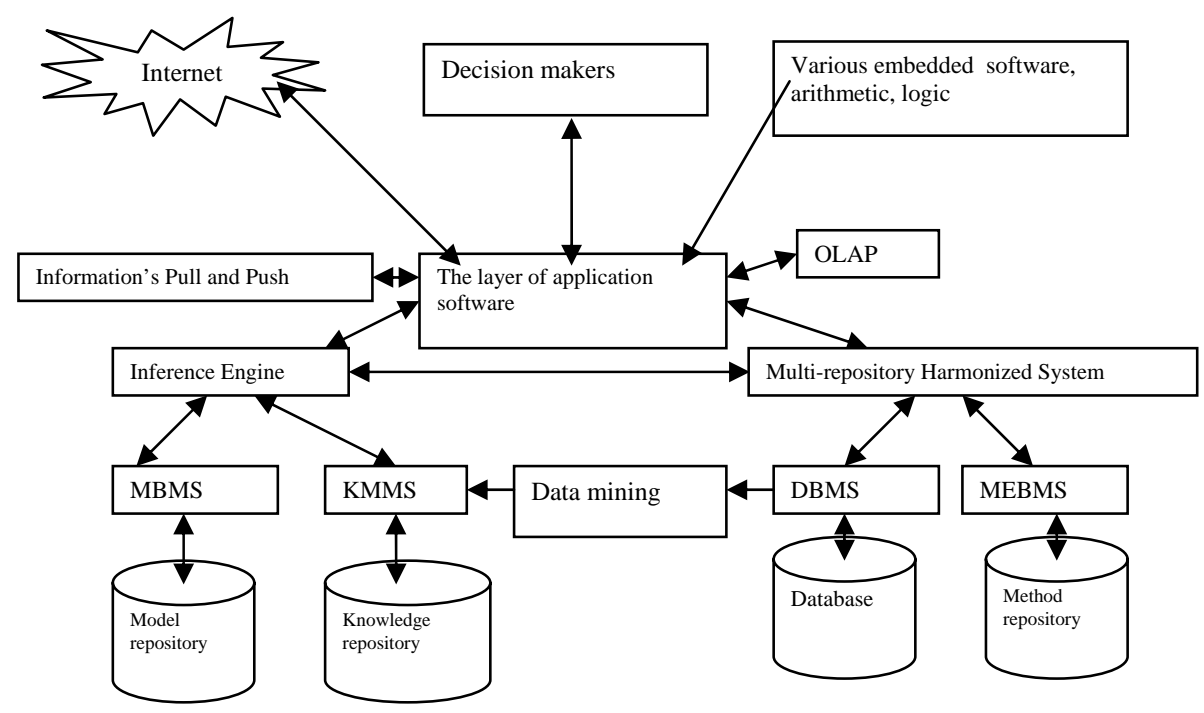

Figure 1: Current BI/DSS model [5]

ability and understanding of the operation of the information system directly contribute to the effectiveness of system use in the decision processes. A customized and easily-understood interface is necessary for users to improve their performances in business decision making. Secondly, the importance of how information collection regarding competitors and markets would relate to the sources of data and the external environment which includes competitors and marketing. Finally, questions are asked regarding the internet access portal and controller, especially the security and speed in data transmission between internal system and external environments. In order to obtain an overview of the perspectives from the six interviewees, a total of 10 questions are discussed during the data collection. The analysis of the findings is summarized below.

\section{Research Findings}

At the beginning of our interviews, the five individuals had to explain how the CI system works and what contributions it makes to their work. The majority of people from the business area as well as from the IT area state that CI should not be considered only the use of intelligence tools. They emphasize that CI is also a blending of culture, content, technology, process, and people into the creation of a market-savvy intelligence function. 
After that we ask how the user ability and understanding of the operation of information systems directly affect the effectiveness of the decision-making process. Our respondents agree that user's feedback is extremely important in the process of implementing IT as well as maintaining simplicity and consistency. Next, we inquire how much the company invests in this system since it has been implemented. They generally indicate that the corporate spending ranges from $1 \%$ to $5 \%$ of its net income per year for the whole IT system. The follow-up question asks about the ratio of investment to return with this system. No concrete figures are given but management believes that the savings from the system are obvious. Finally, we ask if a special user interface has been designed to help users better understand and use the system. We learn that their web-based, user friendly interface helps employees to better understand and use the system.

We also learn that the users are involved in every phase of the CI - from design, information collection and analysis to intelligence, value-added report generation and report presentation. People are considered a vital factor in CI; the people factors impact the efforts in the implementing of the CI systems.

The portal with web-based access for global employees allows all employees to input the information gained from global markets and competitors at the local levels. The information is then categorized and synthesized with a variety of intelligence tools and put in the database of global CI.

Next, the most important step, a range of specialists, from a variety of fields - sale/market, customer/competitor, strategy/development, etc,-work on identifying and analyzing these information, attaching feedbacks and comments into those information based on their understandings and experiences, finally posting and distributing those extracted and identified information back to the web portal to serve the needs of the users at different levels.

It is only after this step that the collected information has passed through the transformation from raw data into business knowledge — the competitive intelligence. "No matter how much money you have spent, if no people is involved in this process, all you get is business information but not competitive intelligence," the response of an IT person to a CIO of another company after hearing how the company just poured millions dollars into upgrading its CI system but did not want to trust people's judgment.
Over 80 percent of corporate America's business knowledge resides in the brains of employees-a huge untapped asset [13, 21].

Furthermore, it is also very important that the reports generated by CI be distributed back to users. If meaningful reports cannot reach the users in a timely and effective manner, all efforts done before will be useless. On the other hand, users' ability and understanding of the system operation can impact directly the effectiveness of system use. As one of the interviewees says, the first important thing in designing the interaction with users is how to present the final result to users. The study only focuses on BIDSS/CI in the area of commercial and business applications. It has not been extended to other fields such as politics or military affairs.

\section{DISCUSSION}

In this research, a description is provided about the BIDSS in current practices as well as the platform and critical components required to deploy and implement BIDSS. In addition, based on the analysis and perspectives from the interviewees, a new BIDSS/CI model is created and reintegrated. The new model identifies at least three problems missing in current studies on BIDSS.

First, the new model highlights information collection from the marketplace and competitors. It separates the information collection into external and internal environments, which is more reasonable and effective for an organization to fully use its limited resources [5]. Secondly, people/user perspective is recognized as a vital factor for the success of any BIDSS/CI because people/user is the source stream of all competitive intelligence. Therefore, people/user must be involved in a BIDSS/CI deployment and implementation, especially in the process of the creation of intelligence information.

Furthermore, this research discovers that an effective BIDSS/CI must consider a suitable way to interact with or respond to people/user abilities and understandings in system implementation. For example, an easy-to-use, friendly interface may bridge the gap between people/user abilities and effectiveness of system use.

Finally, with the need to extract information from internal databases and the internet as well as to transform this collected data into useful knowledge, the system security issue is identified as a necessary functionality in building the new model. The new 
model is embedded with security software in a portal or controller platform for securing the database and retrieving quality information from the external world. All CI package solutions purchased from vendors are required to include security mechanisms. Figure 2 illustrates the new framework for BIDSS with the three levels identified in the BIDSS framework in Figure 1.

First level (the bottom level) is the level of information's collection and storage. A multi repository consists of the data warehouse which functions as the database of data collected and extracted from a variety of sources and waiting to be retrieved and analyzed based on the business goals. The information or data can be from either a firm's internal environment such as an enterprise resource planning (ERP) system, a customer relationship management (CRM) system, a knowledge management (KM) system, or external environment with competitor and market information. This information will be sorted or classified based on certain business requirements and stored in the data warehouse.
The second level (middle level) is the analytical level embedded with a variety of BI/DSS tools. This layer is the core of the system responsible for data analyzing, filtering, refining, etc, and generating reports in response to user queries during the process of decision making. This layer includes various intelligent tools or software such as OLAP, data mining, inference engine, fuzzy logic, and so on. The goal of the layer of applications is to transform raw data gathered from various data sources into useful and valuable business information, and finally into business knowledge.

The third or top level reports, delivers or presents the final results to users. The user - decision maker--has a central role to play in a BIDSS system. His/her ability to use the system efficiently is directly linked to the limit of his/her knowledge of the system. Therefore, a customized and easily-understood user interface is a bridge to fast connect users to diverse functions provided in the system [20]. Thus, this accelerates the process in business decisions and improves the effectiveness of business performances.

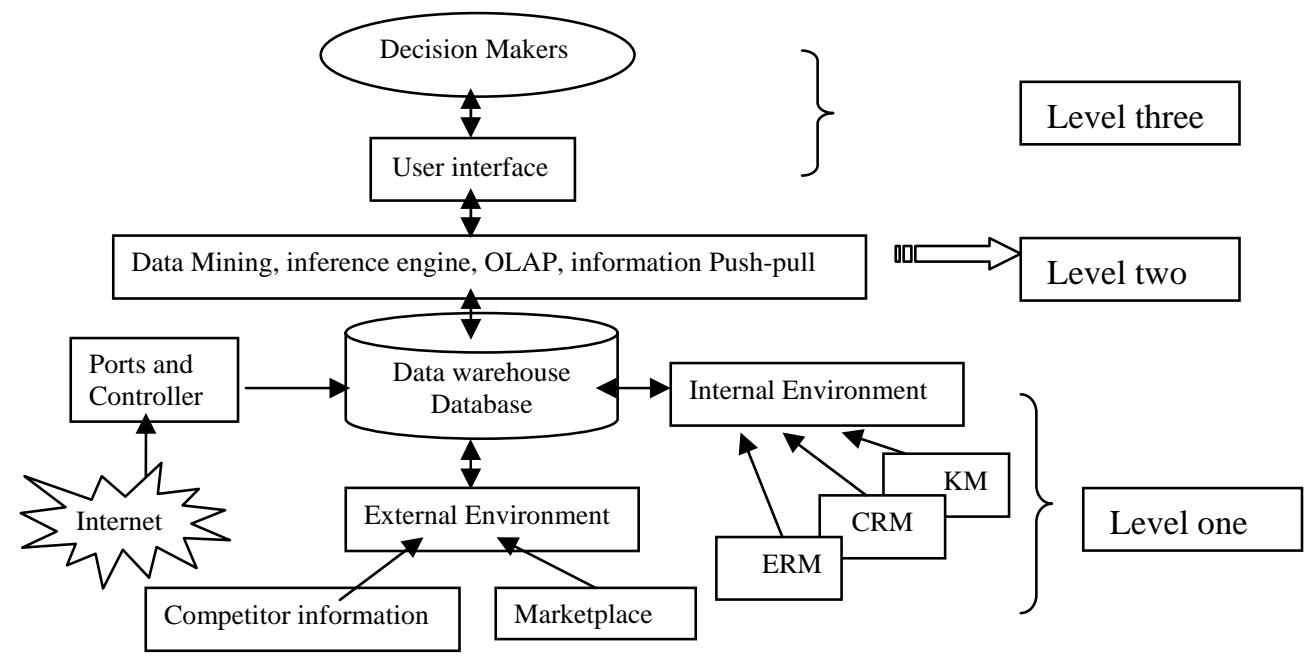

Figure 2: Proposed Framework for BIDSS/CI Systems

Today, with rapidly changing business environment and the age of information explosion, the need for timely and effective business information is recognized as essential for organizations not only to succeed, but even to survive [2]. To gain this advantage from rich business information, an organization needs a powerful and effective IT system to collect, analyzes, synthesize and disseminate these information, and create business knowledge for 
meeting business objectives. BIDSS/CI is designed for that goal. However, many companies invest resources into BIDSS/CI software and hardware, but ignore the impact and role of people/user factors on the effectiveness of deploying and implementing BIDSS/CI. By reviewing the above research findings, the authors believe that in deploying and implementing a BIDSS/CI, the people/user perspective is a critical factor for the success of any kind of BIDSS/CI.

The goal of BIDSS/CI is to transform raw data collected from various sources into business knowledge so as to help people/user make business decisions. Business knowledge is recognized as the creation of competitive intelligence but not the simple extraction of information. BI, DSS or CI software and other intelligence tool package do not really produce competitive intelligence for an organization; instead, they are valuable in helping people/user to collect, organize, and classify the information. Experienced people and users are the only source of competitive intelligence because the competitive intelligence is created only after the feedbacks and comments have been added by those people/users based on their professional experiences and understandings of that information given to them. Therefore, for the system to interact with people/user, some considerations about people/user's knowledge and understandings on the system need to be concerned.

People/user knowledge and understanding determine behaviors, preferences and habits in the system use. To an extent, these may impact the effectiveness of a BIDSS/CI use. Thus many use-friendly functions to enhance interactions with users are needed. Applied procedures, ways of information processing and exchange, and especially human factors, can determine behaviors, preferences and habits in any system use. In fact, it could be that the use of BIDSS/CI systems is highly personalized. For example, a "NewsFeed", an agile function embedded in the email system, can automatically offers and disseminates a list of brief news headings relevant to markets or competitors to different level business people by email. Business people then can click headings to link to the corporate CI databases to retrieve relevant information that has been value-added, intelligence-added by the global CI analytic team to correspondingly react to changes in the markets and activities of competitors. However, ultimately, the decisions made are based on the users' perspectives, knowledge, and experiences. Email is the most common and familiar communication tool for every employee today. The CI team in designing such a simple portal for its users via "NewsFeed" fully correlates the users' knowledge and understandings with performance efforts of system use. Furthermore, users also benefit because this greatly reduces time needed to search for information. Without an understanding of user's abilities, intelligence needs, preferences, and behaviors, even the most elaborate, feature-rich offering will fall short because it would not get used and would not benefit its intended users as well [13].

\section{CONCLUSION}

BIDSS/CI can be defined as a seamless integration of a series of computerized systems, software and people/user's activities and minds that are involved in the BIDSS/CI process [10]. Its goal is to provide timely and accurate business knowledge - competitive intelligence for decision makers so as to help them make business decisions, providing competitive intelligence edge for an organization. A BIDSS/CI can be divided into three phases: the information collection and repository phase; software application and data analysis phase; and use interface.

The first phase is the collection of the data and the storage of the information in appropriate databases. The second phase is the core of a BIDSS/CI. In this people/user phase, the people/user identifies the potential value for those extracted information from the first phase, creates value-add and intelligence information by attaching their feedbacks and comments according to their experiences as well as business knowledge. In the third phase, when designing the interaction between system and users, the most important thing is to think about a way to get CI information back to users. For example, how is the report presented or delivered to users, or how can users quickly get CI information from the system? Due to the limitations of users' abilities and understanding of the system when designing a portal or interface for interacting with users, those constraints have to be considered. Once an organization really understands that the power of the BIDSS/CI 
comes from the people/user efforts, it is relatively easy to reap the benefits that the BIDSS/CI brings to the organization.

The limitations of this case study require that further research should be conducted to analyze the human aspects of the enhanced use of BIDSS/CI systems. At the same time, the authors acknowledge that a series of economic facts are not recorded or built into BIDSS/CI systems, but are clearly used in managerial decision making. In fact, competitive factors used in such decision making would not be made available to the researchers as they could be considered as issues of strategic competitiveness that are proprietary to the organization.

\section{REFERENCES}

1. Abukari, K \& Vijay, J. (2003). Business intelligence in action. CMA Management. 77(1), 4-15.

2. Antti, L \& Virpi, P, (2006). The measurement of business intelligence. Information System Management. 23(1), 3241.

3. Bauerschmidt, A. \& Bloodgood, J. M. (2002). Competitive Analysis: Do managers accurately compare their firms to competitors? Journal of Managerial. 14 (4), 418.

4. Bouaka, N and David, A. (2004). A proposal of a decision-maker problem for a better understanding of informational needs. 1st International Conference on Information \& Communication Technologies: from theory to Applications - ICTTA'04. (Damas, Syrie), 5p.

5. Cao, P. (2002). The theory and application of business intelligence. Journal of Information Science of Beijing University. 101(3), 15-21.

6. Cody, W. F. \& Kreulen, J. T. (2005). Competitor analysis and its defenses in the E-marketplace. Communication of the ACM. 48(8).

7. David C. T. \& Kerr, I. (2004). Cultivating Your Cultural Intelligence: Before Business Professional Do Business with Other Cultures, They Must Know How to Bridge the Cultural Divide. Security Management. 48(8), 30.

8. David, F. R. \& Groom, J. R. (2001). Competitive intelligence activity among
Small Firms. SAM Advanced Management Journal. 66, 12.

9. Hayen, R. L., \& Holmes, M.C. (2006). Decision Support Systems: An Investigation of Characteristics. Business Research Yearbook. 13, 117-122.

10. Haynes, S. R. (2001). Explanation in Information System. A Design Rationale Approach. PhD thesis submitted to The London School of Economics.

11. Kreulen, J. T. (2002). The integration of business intelligence and knowledge management. IBM Systems Journal. 41(4), 697-713.

12. Len, D. (2005). Ten Steps to Total Data Quality. Business intelligence Journal. 10(2), 57-63.

13. Li, Z. H. (2003). The analysis and application of Business intelligence technology. Journal of JiLin University. 21(1).

14. Outsell. (2001). Information about information - The Making of a High-Impact Market Intelligence Function. 4 (16).

15. Sam, E. (2005, February). Is intelligence in the wrong hands. Security management. 49, 124.

16. Su, Z. \& Jiang, J. (2004). Market intelligence: An entity-based system for managing market intelligence. IBM Systems Journal. 43(3), 534-545.

17. Thierauf, R. J. (2001). In Effective Business Intelligence Systems. Quorum Books. 65-94.

18. Vickil, S. \& Donna, F. (2005). Database for Advances in Information Systems. Competitive Intelligence Systems: Qualitative DSS for Strategic Decision Making. New York: Spring 2005. 36(2), 4358.

19. Watson, et.al. (2006). Real-Time Business Intelligence: Best Practices At continental Airlines. Information System Management. 23(1), 7-22.

20. Xie, H, I. (2002). Patterns between interactive intentions and informationseeking strategies. Information Processing \& Management. 38 (1), 55-77.

21. Yoon, V, et, al., (2005). Using Agent Technology for Company Knowledge Management. Information Resources Management Journal. 18 (2), 94-114. 